\title{
Supermassive Black Holes in Galaxies
}

\author{
Amy J. Barger ${ }^{1,2,3}$ \\ ${ }^{1}$ Department of Astronomy, University of Wisconsin-Madison, \\ 475 N. Charter St., Madison, WI 53706, USA \\ email: barger@astro.wisc.edu \\ ${ }^{2}$ Department of Physics and Astronomy, University of Hawaii, \\ 2505 Correa Road, Honolulu, HI 96822, USA \\ ${ }^{3}$ Institute for Astronomy, University of Hawaii, \\ 2680 Woodlawn Drive, Honolulu, HI 96822, USA
}

\begin{abstract}
Chandra and $X M M$ detect X-rays emitted during accretion onto supermassive black holes, even when they are highly obscured. I review what has been learned about the cosmic evolution of the X-ray luminosity functions and the reconstruction of the accretion history of supermassive black holes from extensive follow-up observations of both deep and wide-area Chandra X-ray surveys.
\end{abstract}

Keywords. cosmology: observations, galaxies: active, galaxies: distances and redshifts, galaxies: evolution, X-rays: galaxies.

\section{Introduction}

In order to understand how supermassive black holes form and evolve, we need to determine the accretion history of the universe. However, much of the accretion power in the universe is absorbed, making it difficult to measure at optical wavelengths. The Chandra and XMM X-ray observatories have revolutionized distant active galactic nuclei (AGNs) studies. It is now possible to map the history of the AGN population using hard $\mathrm{X}$-ray surveys and to compare high and low-redshift samples chosen in the same restframe 2-8 keV band. Hard X-rays have the advantage in that they can directly probe AGN activity, are uncontaminated by star formation processes at the X-ray luminosities of interest, and detect all but the most absorbed sources.

The advantage of Chandra for this kind of work is its high positional accuracy, which minimizes misidentifications of faint optical galaxy counterparts. It is important to note, however, that even with a $2^{\prime \prime}$ match radius, about $20 \%$ of the faint-end optical identifications ( $R=24-26)$ of the Chandra sources will be spurious (Barger et al. 2003), while with the $X M M$ data, the percentage of misidentifications will be far worse. Moreover, unlike $X M M$, Chandra does not reach the confusion limit, even with the deepest observations.

\section{Chandra X-ray Data}

The two deepest images of the X-ray sky ever taken are the 2 Ms Chandra Deep Field-North (CDF-N; Alexander et al. 2003) and the $1 \mathrm{Ms}$ Chandra Deep Field-South (CDF-S; Giacconi et al. 2002). These data resolve greater than 80 to $90 \%$ of the $2-8 \mathrm{keV}$ $\mathrm{X}$-ray background (XRB) into discrete sources. The CDF-N exposure samples a large, distant cosmological volume down to very faint X-ray flux limits of $f_{2-8 \mathrm{keV}} \approx 1.4 \times$ $10^{-16}$ ergs $\mathrm{cm}^{-2} \mathrm{~s}^{-1}$ and $f_{0.5-2 \mathrm{keV}} \approx 1.5 \times 10^{-17} \mathrm{ergs} \mathrm{cm}^{-2} \mathrm{~s}^{-1}$. The CDF-S exposure is only a factor of two shallower, but this is still substantially fainter than the X-ray flux of the sources that contribute the most to the XRB (Cowie et al. 2002; see Fig. 1). 


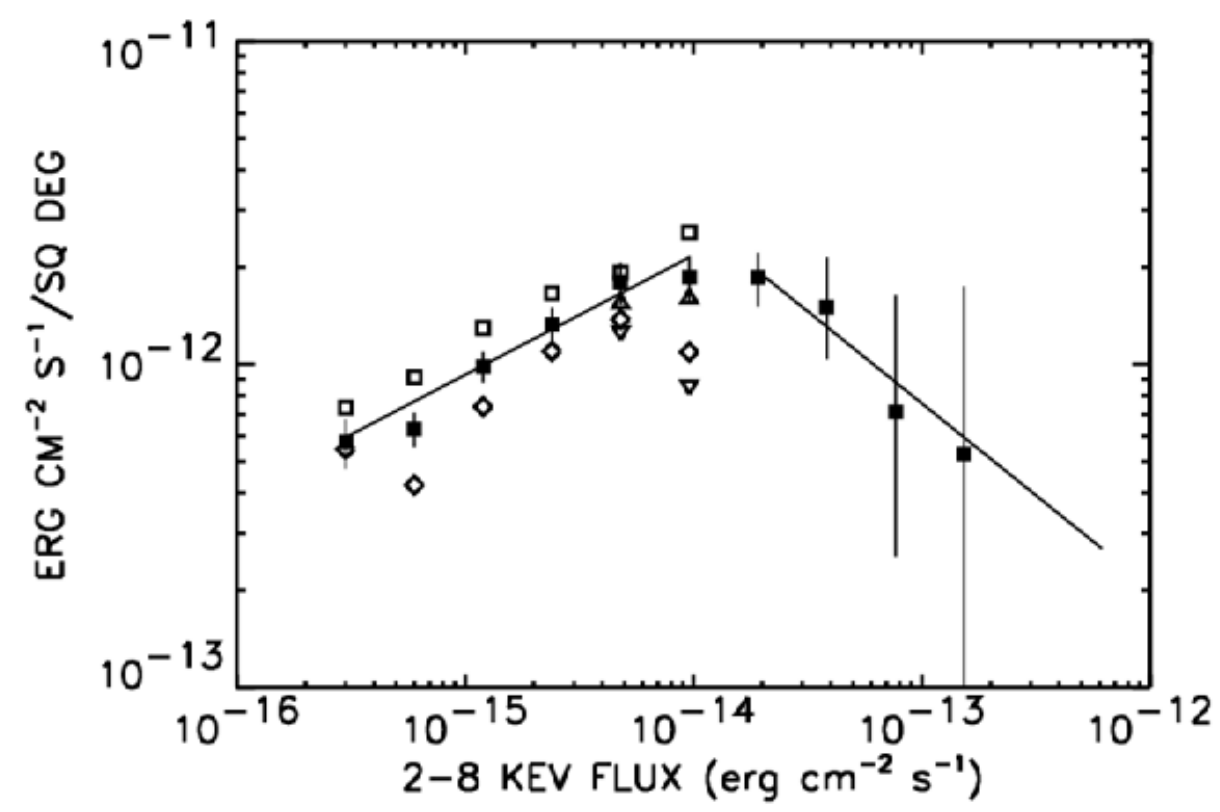

Figure 1. Contribution to the XRB vs. $2-8 \mathrm{keV}$ flux for individual fields (shown only below $10^{-14} \mathrm{ergs} \mathrm{cm}^{-2} \mathrm{~s}^{-1}$, where the error bars are small) and the combined sample (solid squares). Lines show power law fits from Cowie et al. (2002). Figure courtesy of Cowie et al. (2002).

An unexpected result from the Chandra deep field observations was the substantial field-to-field variations observed in the X-ray number counts on the scale of a single Chandra image (see Fig. 1). The origin of this variance is not known, but it is likely due to cosmic variance originating in large scale structure. Thus, many Chandra fields are needed to get the average true number counts. Large area surveys are also needed to get the necessary volume to map the low-redshift luminosity function and to sample the small number of very high-redshift sources. This can be done either with serendipitous pointings (the ChaMP survey strategy) or with contiguous fields. The advantage of the contiguous fields approach is that one can then also study hard X-ray source clustering.

There are a number of ongoing wide-area Chandra and XMM surveys (e.g., the ChaMP survey, the Groth Strip survey, the Extended CDF-S, the Wilkes Lockman Hole field, and the COSMOS survey). However, the only current survey with a very high level of spectroscopic identifications is the Chandra Large Area Synoptic X-ray Survey (CLASXS), which consists of nine overlapped Chandra ACIS-I pointings in the Lockman Hole Northwest field covering $\sim 0.4$ square degrees to X-ray flux limits of $f_{2-8 \mathrm{keV}} \approx 3 \times 10^{-15}$ and $f_{0.5-2 \mathrm{keV}} \approx 5 \times 10^{-16}$ ergs $\mathrm{cm}^{-2} \mathrm{~s}^{-1}$ (Yang et al. 2004).

Yang et al. (2003) found that the full range of cosmic variance seen in the earlier, individual Chandra pointings was represented in the number counts of the nine CLASXS pointings. They found the clustering in both the hard and soft X-ray bands to be significant, though, interestingly, the X-ray sources detected only in the hard band appeared to be substantially more clustered. This means that the Chandra hard X-ray selected sources probably have the largest correlation strength of any AGN population, one that 
is similar to that of massive galaxies. Indeed, in the CDF-N, the hard X-ray AGNs lie in the same density environments as massive galaxies (Cowie et al. 2004).

\section{Spectroscopic Completeness}

We supplement the CLASXS, CDF-N, and CDF-S surveys with the very high-luminosity $A S C A$ sample of Akiyama et al. (2000). Figure 2 shows the useful flux ranges of the hard X-ray samples in these fields. Above $f_{2-8 \mathrm{keV}} \sim 10^{-14}$ ergs $\mathrm{cm}^{-2} \mathrm{~s}^{-1}, 80-90 \%$ of the hard X-ray sources have redshifts, while below this flux, $\sim 60 \%$ do. The fact that the fraction of sources with spectroscopic identifications drops at fluxes below $f_{2-8 \mathrm{keV}} \sim 10^{-14}$ ergs $\mathrm{cm}^{-2} \mathrm{~s}^{-1}$, which is the same flux where the contributions to the hard XRB peak, is a consequence of the fraction of broad-line AGNs being much higher at the brighter X-ray luminosities, as well as the fainter X-ray sources being optically fainter.

The spectroscopic samples are highly complete to $R=24.5$, and the use of photometric redshifts increases the overall identified fraction to about $85 \%$. The spectroscopic incompleteness at $z>1.2$ is due to the absence of strong features in the spectra and the faintness of sources at these redshifts. We classified the spectroscopically identified X-ray sources into four optical spectral classes (absorbers, star formers, high-excitation sources, and broad-line AGNs). Hereafter, I refer to all sources that do not show broadline $\left(\mathrm{FWHM}>2000 \mathrm{~km} \mathrm{~s}^{-1}\right)$ signatures as optically-narrow AGNs. We find that the broad-line AGNs are nearly all soft and show essentially no visible absorption in Xrays, while the optically-narrow AGNs are well-described by a power-law spectrum with photoelectric absorption spread over a wide range of $N_{H}$ values. Thus, it is possible to separate roughly the broad-line AGNs and the optically-narrow AGNs on the basis of X-ray colors alone (e.g., Szokoly et al. 2004), without knowing the optical spectra. However, there will be a small amount of contamination from stars, from the small number of optically-narrow AGNs that have soft X-ray colors, and from the small number of broad-line AGNs that have hard X-ray colors.

\section{Evolution of the Hard X-ray Luminosity Function}

We define the hard X-ray luminosity function (HXLF) versus rest-frame $2-8 \mathrm{keV}$ $\mathrm{X}$-ray luminosity and redshift $\left[d \Phi\left(L_{X}, z\right) / d \log L_{X}\right]$ as the number of X-ray sources per unit comoving volume per unit base 10 logarithmic luminosity that lie in the redshift interval. With the advent of the Chandra and XMM data, there have been a number of computations of the evolution of the HXLF with redshift (e.g., Cowie et al. 2003; Steffen et al. 2003; Ueda et al. 2003; Hasinger 2003; Fiore et al. 2003; Barger et al. 2005), while Sazonov \& Revnivtsev (2004) have used the RXTE data to compute the local 3-20 keV luminosity function. The latest HXLF determinations by Barger et al. (2005) use only the spectroscopically identified X-ray sources with $L_{2-8} \mathrm{keV}=10^{42} \mathrm{ergs} \mathrm{s}^{-1}$ in the CDF-N, CDF-S, and CLASXS fields. They assume that any source more luminous than $L_{2-8} \mathrm{keV}=10^{42} \mathrm{ergs} \mathrm{s}^{-1}$ is very likely to be an AGN on energetic grounds (Zezas et al. 1998; Moran et al. 1999).

The relative HXLFs of the total and the broad-line AGNs from Barger et al. (2005) reproduce the results of Steffen et al. (2003), who showed that the dominant population at these higher X-ray luminosities, where the redshift identifications are very complete, is broad-line AGNs, while at the lower X-ray luminosities, the non-broad-line AGNs dominate (hereafter, I refer to this as the Steffen effect). Since broad-line AGNs are 


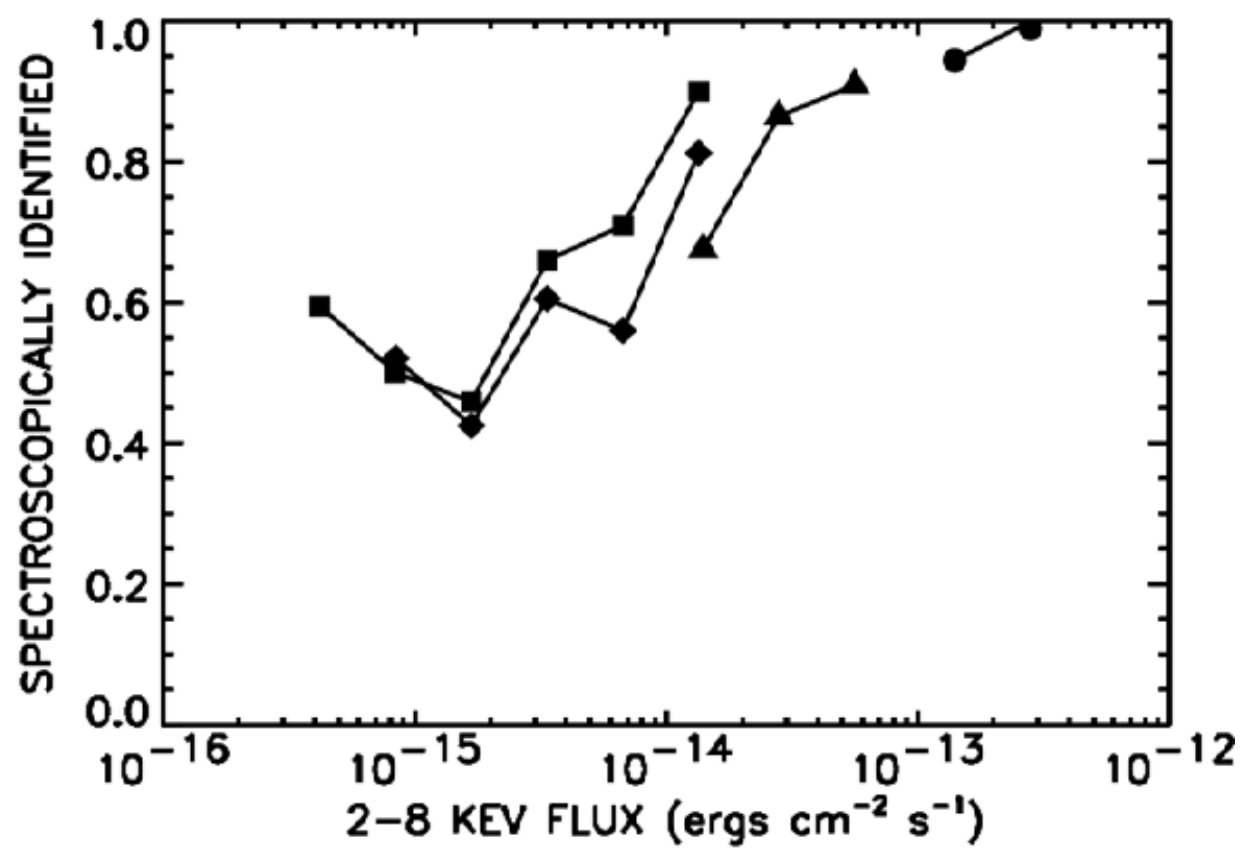

Figure 2. Spectroscopic completeness (i.e., the fraction of observed sources that are spectroscopically identified) of the hard X-ray surveys that make up the total hard X-ray sample (squares - CDF-N, Barger et al. 2003; diamonds - CDF-S, Szokoly et al. 2004; triangles CLASXS, Steffen et al. 2004; circles - ASCA, Akiyama et al. 2003). Sources are grouped into flux bins that increase by a multiplicative factor of 2 . Only flux bins containing more than 10 sources are plotted to illustrate the useful flux ranges of the various samples. Figure courtesy of Barger et al. (2005).

straightforward to identify spectroscopically, and the bulk of the X-ray sources at these luminosities have now been observed, one does not need to worry that broad-line AGNs are making up a substantial fraction of the unidentified population. Since these results suggest a luminosity dependence in optical spectral type, the simple unified model for AGNs, in which there is no luminosity or redshift dependence of the obscuration, does not appear to be valid.

Figure 3 shows the measured HXLFs from Barger et al. (2005) for two low redshift intervals (open squares $-z=0.2-0.4$; solid diamonds $-z=0.8-1.2$ ). Poissonian $1 \sigma$ uncertainties are based on the number of galaxies in each luminosity bin. For these redshifts, the HXLFs were computed from observed-frame $2-8 \mathrm{keV}$. An intrinsic $\Gamma=$ 1.8 was assumed, for which there is only a small differential $K$-correction to rest-frame $2-8 \mathrm{keV}$. The $z=0 \mathrm{HXLF}$ from Sazonov \& Revnivtsev (2004)'s RXTE analysis is also shown (dotted line).

The redshift data for the Chandra sources give a remarkable picture of the evolution of the AGN population to $z \sim 1.2$. The HXLF undergoes a steep pure luminosity evolution (PLE), with the characteristic luminosity evolving as $(1+z)^{3.2}$ out to $z \sim 1.2$ (see Fig. 3 ). The same PLE model describes the luminosity function of both the broad-line AGNs alone and all the hard X-ray sources together, though the two samples have very different 


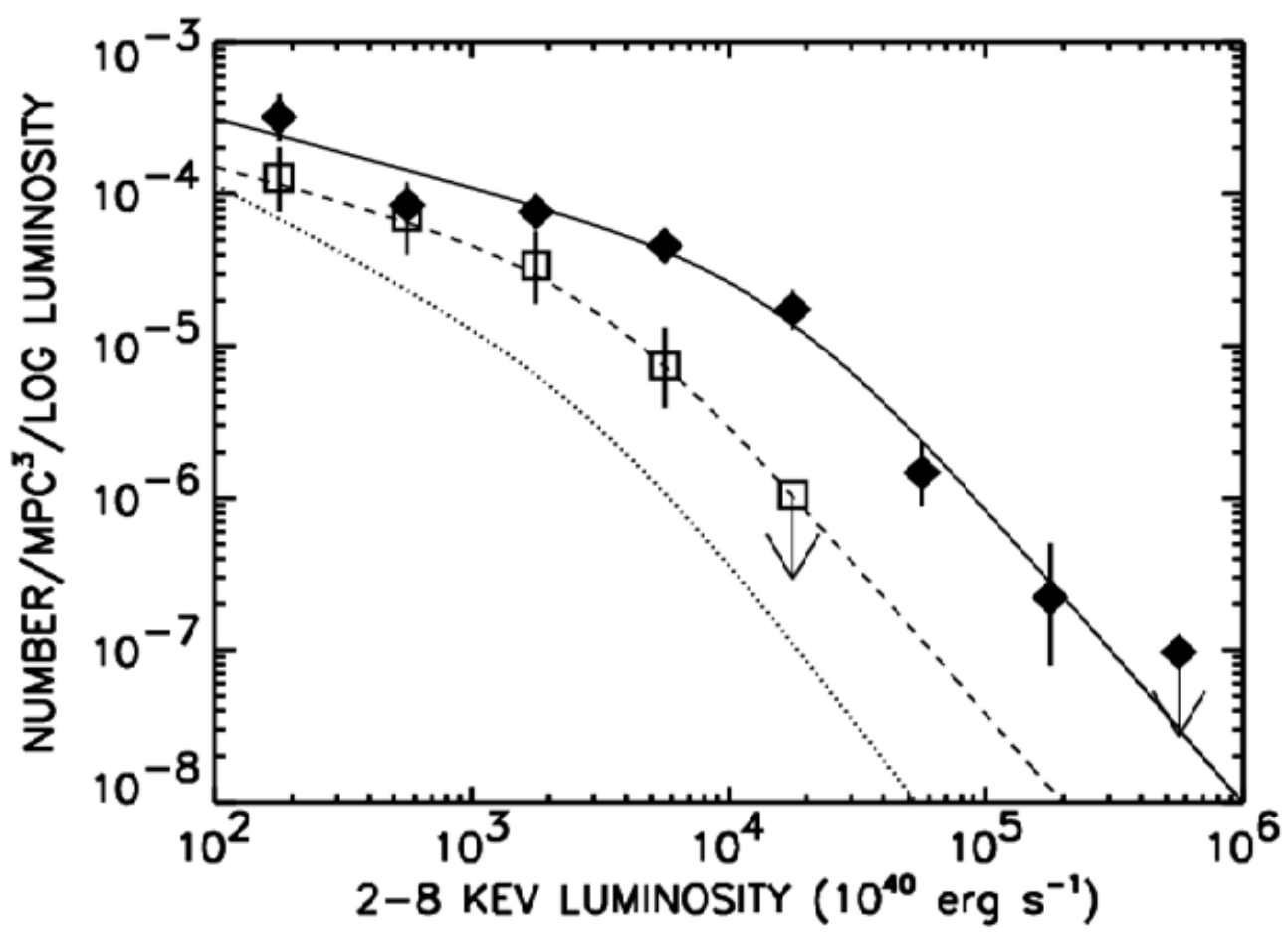

Figure 3. Rest-frame $2-8 \mathrm{keV}$ luminosity function per unit logarithmic luminosity at $z=0$ (dotted curve - Sazonov \& Revnitsev's 2004 RXTE analysis), $z=0.2-0.4$ (open squares), and $z=0.8-1.2$ (solid diamonds). Solid curve is a double power-law fit to the $z=0.8-1.2$ total HXLF. Dashed curve shows a pure luminosity evolution model where only the characteristic luminosity evolves as $(1+z)^{3.2}$ over the $z=0-1$ redshift interval. This model also fits the local $R X T E$ determination.

luminosity functions. This evolution also matches to the recent determination of the local HXLF by Sazonov \& Revnivstev (2004). At the higher redshifts, incompleteness is potentially a larger source of error because of the difficulty with measuring the host galaxy redshifts in those intervals. However, even with maximal incompleteness corrections, the HXLFs at $L_{2-8} \mathrm{keV}<10^{44} \mathrm{ergs} \mathrm{s}^{-1}$ lie below the maximum likelihood fits to the $z=0-1.2 \mathrm{HXLF}$ computed at $z=1$, suggesting a peak in the universal AGN energy density production rate near $z=1$.

Correspondingly, the production rate of the AGN radiation drops rapidly, as is shown in Figure 4. This is based on direct summation, but integration of power-law fits to the HXLFs give a similar answer. The largest uncertainty is the redshift distribution of the unidentified sources, rather than the small effects of extrapolation outside the observed luminosity range. However, even with this incompleteness uncertainty, the energy density production rate is at most flat beyond $z=1$, and, more realistically, is slightly falling.

From Figure 4, we see that the dominant period of supermassive black hole production is at redshifts near $z=1$. This is very different than what had been expected preChandra. Such an extremely rapid evolution with redshift bears a striking resemblence to the overall redshift evolution of the star formation rate density in $z<1$ galaxies (e.g., Wilson et al. 2002). We also see from Figure 4 that at $z<1.5$, most of the hard X-ray energy density production is due to optically-narrow AGNs. This highlights the 


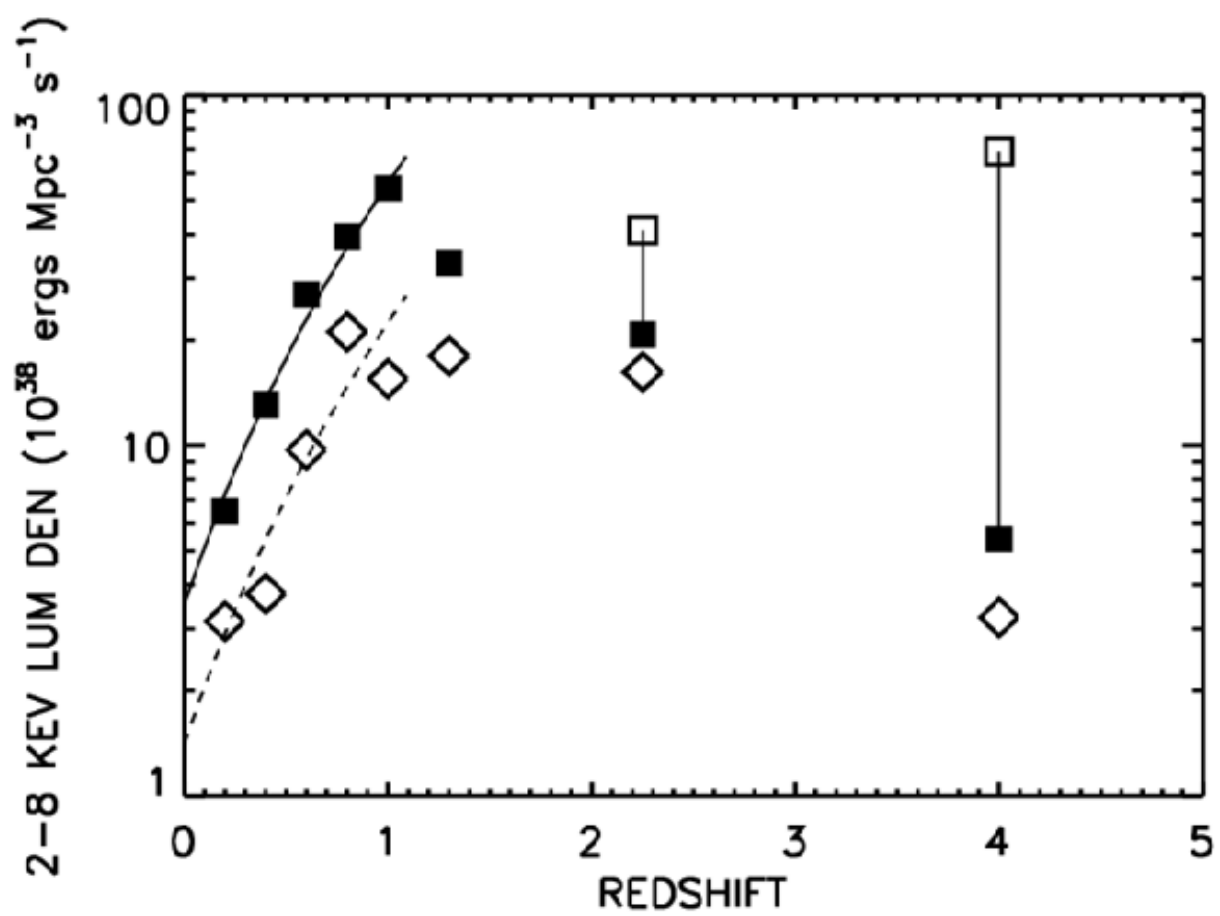

Figure 4. Evolution with redshift of the rest-frame $2-8 \mathrm{keV}$ comoving luminosity density production rate of $L_{2-8 \mathrm{keV}}>10^{42} \mathrm{ergs} \mathrm{s}^{-1}$ sources. Solid squares (open diamonds) denote the measured values for the total sample (broad-line AGNs only). Open squares show the upper limits found by assigning all of the unidentified sources to the centers of each redshift bin. Solid and dashed curves show a $(1+z)^{3.2}$ evolution over the $z=0-1.2$ range. Figure courtesy of Barger et al. (2005).

importance of using X-ray data to identify obscured sources and then including them in the determination of the accretion history of the universe.

We now return to the interesting observation that broad-line AGNs dominate the number densities at the higher X-ray luminosities, while non-broad-line AGNs dominate at the lower X-ray luminosities (the Steffen effect). Could the absence of broad-line AGNs at low X-ray luminosities be explained if the nuclear UV/optical light of optically-narrow AGNs were being swamped by the host galaxy light? That is, might galaxy dilution (see, e.g., Moran et al. 2002) be a partial explanation of the Steffen effect? With the highresolution ACS GOODS-North data (Giavalisco et al. 2004), Barger et al. (2005) were able to separate the nuclear component of each source from the host galaxy light, even at the higher redshifts, in order to analyze the nuclear colors. It is well-known that the nuclear UV magnitudes and the X-ray fluxes for broad-line AGNs are strongly correlated. Thus, if the galaxy dilution hypothesis were correct, we would expect the opticallynarrow AGNs to be similarly correlated when we isolate their nuclear UV/optical light. In Figure 5, we see that that turns out not to be the case. Instead, the nuclei of the optically-narrow AGNs are much weaker relative to their X-ray light than are the broadline AGNs. Thus, the absence of broad-line AGNs at low X-ray luminosities is not a dilution effect. Optically-narrow AGNs really have weaker UV/optical nuclei relative to the X-rays. Now that galaxy dilution has been ruled out as a partial explanation of the 


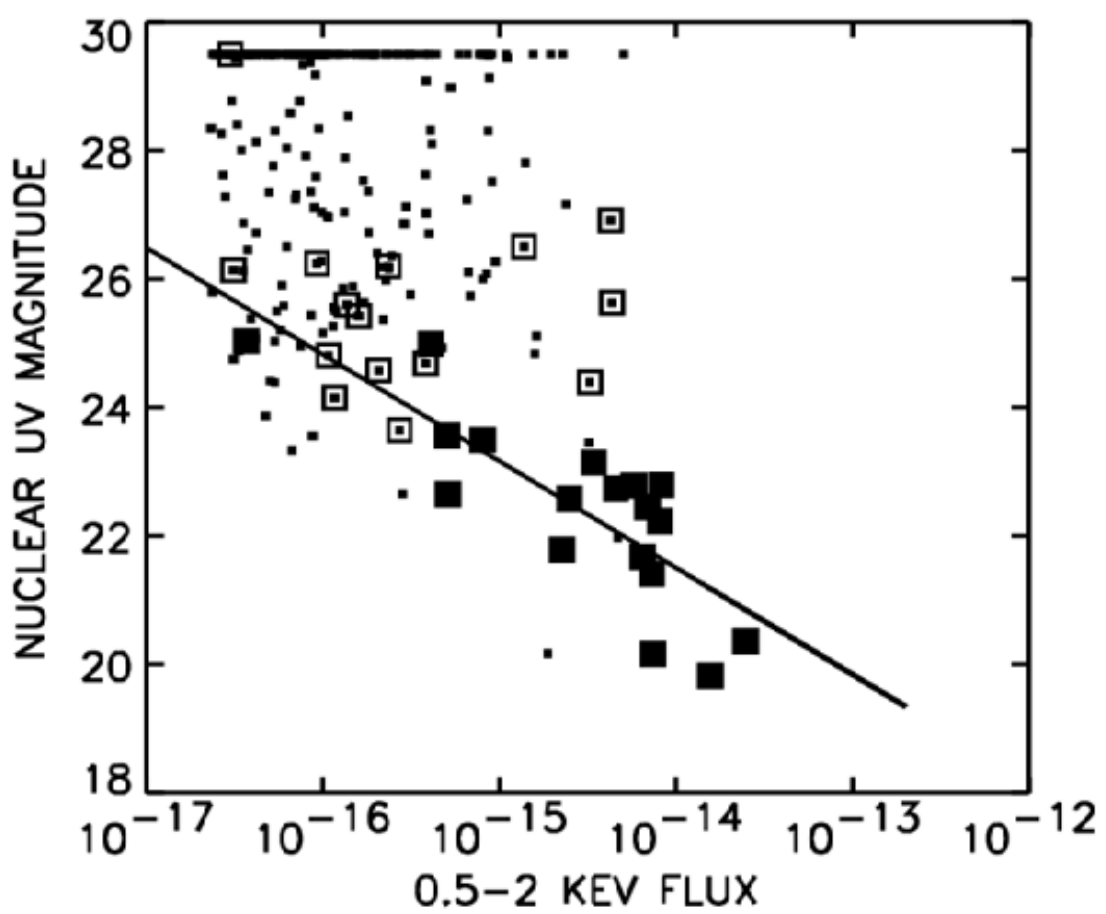

Figure 5. Nuclear UV magnitude vs. $0.5-2 \mathrm{keV}$ flux, showing that there is a strong correlation between the two for broad-line AGNs. Broad-line AGNs (optically-narrow AGNs) are denoted by large (small) squares. High-excitation sources are enclosed in large open squares. Sources with no measurable nuclei (all but one of these are spectrally classified as normal galaxies) are shown at a nominal magnitude of 29.5 , which is roughly the $2 \sigma$ limit for the small $0.3^{\prime \prime}$ diameter aperture used to measure the nuclear magnitude. Solid line shows a linear relation fitted to the broad-line AGNs. Figure courtesy of Barger et al. (2005).

Steffen effect, it can be stated firmly that the simple unified model has failed because of the existence of the Steffen effect. The simplest interpretation of the Steffen effect is that the covering fraction of obscuration in the AGNs is extremely small at high X-ray luminosities (so that all we can see at these high luminosities are broad-line AGNs) and extremely large (near unity) at low X-ray luminosities (so we do not see broad-line AGNs at these low luminosities).

\section{Conclusions}

Optically-selected AGNs (otherwise known as broad-line AGNs) are only a subset of Xray selected AGNs. AGNs evolve very rapidly to $z \sim 1.2$, consistent with pure luminosity evolution. The observation that there are almost no low X-ray luminosity, broad-line AGNs is not a galaxy dilution effect, ruling out the simple unified model. The Barger et al. (2005) hard X-ray luminosity function determinations of the supermassive black hole mass densities are a factor of almost two lower than what Yu \& Tremaine (2002) found due to the $\mathrm{Yu} \&$ Tremaine extrapolation of the optical QSO luminosity function outside the optically observed luminosity range. Comparing this result with the local 
supermassive black hole mass density, there is now room for obscured accretion by the optically-narrow AGNs.

\section{Acknowledgements}

I thank the conference organizers for inviting me to participate in this stimulating meeting. I gratefully acknowledge support from NASA grants CXC GO2-3191A and HST-GO-09425.30-A from the Space Telescope Science Institute, from NSF grant AST 02-39425, and from the Alfred P. Sloan Foundation and the David and Lucile Packard Foundation.

\section{References}

Akiyama, M, Ohta, K., Yamada, T., Kashikawa, N., Yagi, M., Kawasaki, W., Sakano, M., Tsuru, T., Ueda, Y., Takahashi, T., Lehmann, I., Hasinger, G., \& Voges, W. 2000, Astrophys. J. 532,700

Alexander, D.M., Bauer, F.E., Brandt, W.N., Schneider, D.P., Hornschemeier, A.E., Vignali, C., Barger, A.J., Broos, P.S., Cowie, L.L., Garmire, G.P., Townsley, L.K., Bautz, M.W., Chartas, G., \& Sargent, W.L.W. 2003, Astron. J. 126, 539

Barger, A.J., Cowie, L.L., Capak, P., Alexander, D.M., Bauer, F.E., Fernandez, E., Brandt, W.N., Garmire, G.P., \& Hornschemeier, A.E. 2003, Astron. J. 126,632

Barger, A.J., Cowie, L.L., Mushotzky, R.F., Yang, Y., Wang, W.-H., Steffen, A.T., \& Capak, P. 2005, Astron. J. 129, 578

Cowie, L.L., Barger, A.J., Bautz, M.W., Brandt, W.N., \& Garmire, G.P. 2003, Astrophys. J. $584, \mathrm{~L} 57$

Cowie, L.L., Barger, A.J., Hu, E.M., Capak, P., \& Songaila, A. 2004, Astron. J. 127, 3137

Cowie, L.L., Garmire, G.P., Bautz, M.W., Barger, A.J., Brandt, W.N., \& Hornschemeier, A.E. 2002, Astrophys. J. 566, L5

Fiore, F., Brusa, M., Cocchia, F., Baldi, A., Carangelo, N., Ciliegi, P., Comastri, A., La Franca, F., Maiolino, R., Matt, G., Molendi, S., Mignoli, M., Perola, G. C., Severgnini, P., \& Vignali, C. 2003, Astron. ES Astrophys. 409, 79

Giacconi, R., Zirm, A., Wang, J. X., Rosati, P., Nonino, M., Tozzi, P., Gilli, R., Mainieri, V., Hasinger, G., Kewley, L., Bergeron, J., Borgani, S., Gilmozzi, R., Grogin, N., Koekemoer, A., Schreier, E., Zheng, W., \& Norman, C. 2002, Astrophys. J. Suppl. Ser. 139, 369

Giavalisco, M., Ferguson, H. C., Koekemoer, A. M., et al. 2004, Astrophys. J. 600, L93

Hasinger, G. 2003, in: S. S. Holt \& C. Reynolds (eds.), AIP Conf. Proc. 666 The Emergence of Cosmic Structure (Melville: AIP), p. 227

Moran, E. C., Filippenko, A. V., \& Chornock, R. 2002, Astrophys. J. 579, L71

Moran, E. C., Lehnert, M. D., \& Helfand, D. J. 1999, Astrophys. J. 526, 649

Sazonov, S. Yu \& Revnivtsev, M. G. 2004, Astron. \& Astrophys. 421, L21

Steffen, A.T., Barger, A.J., Capak, P., Cowie, L.L., Mushotzky, R.F., \& Yang, Y. 2004, Astron. J. 128,1483

Steffen, A.T., Barger, A.J., Cowie, L.L., Mushotzky, R.F., \& Yang, Y. 2003, Astrophys. J. 596, L23

Szokoly, G.P., Bergeron, J., Hasinger, G., Lehmann, I., Kewley, L., Mainieri, V., Nonino, M., Rosati, P., Giacconi, R., Gilli, R., Gilmozzi, R., Norman, C., Romaniello, M., Schreier, E., Tozzi, P., Wang, J. X., Zheng, W., \& Zirm, A. 2004, Astrophys. J. Suppl. Ser. 155, 271

Ueda, Y., Akiyama, M., Ohta, K., \& Miyaji, T. 2003, Astrophys. J. 598, 886

Wilson, G., Cowie, L. L., Barger, A. J., \& Burke, D. J. 2002, Astron. J. 124, 1258

Yang, Y., Mushotzky, R. F., Barger, A.J., Cowie, L.L., Sanders, D.B., \& Steffen, A.T. 2003, Astrophys. J. 585, L85

Yang, Y., Mushotzky, R.F., Steffen, A.T., Barger, A.J., \& Cowie, L.L. 2004, Astron. J. 128, 1483

Yu, Q. \& Tremaine, S. 2002, Mon. Not. Royal Astron. Soc. 335, 965

Zezas, A. L., Georgantopoulos, I., \& Ward, M. J. 1998, Mon. Not. Royal Astron. Soc. 301, 915 


\section{Discussion}

ZEZAS: Do you find significant differences between the number of sources in the 9 fields of the CLASXS survey?

BARGER: Yes, the 9 fields cover the full range from the CDF-S number counts to the CDF-N number counts.

MirABEL: Could you comment how Chandra results compare with the results from MidIR, submillimetre, and radio?

BARGER: It is true that both the AGN and the star formation histories are changing in a parallel fashion. This is not a coincidence and is probably a consequence of changes in the gas history of galaxies. 